\title{
Immune System Disorder
}

National Cancer Institute

\section{Source}

National Cancer Institute. Immune System Disorder. NCI Thesaurus. Code C3507.

A disorder resulting from an abnormality in the immune system. 